PROCEEDINGS OF THE

AMERICAN MATHEMATICAL SOCIETY

Volume 140, Number 9, September 2012, Pages 3067-3082

S 0002-9939(2011)11176-6

Article electronically published on December 30,2011

\title{
ON INTEGRABILITY OF NONAUTONOMOUS NONLINEAR SCHRÖDINGER EQUATIONS
}

\author{
SERGEI K. SUSLOV
}

(Communicated by Ken Ono)

\begin{abstract}
We show, in general, how to transform the nonautonomous nonlinear Schrödinger equation with quadratic Hamiltonians into the standard autonomous form that is completely integrable by the familiar inverse scattering method in nonlinear science. Derivation of the corresponding equivalent nonisospectral Lax pair is also outlined. A few simple integrable systems are discussed.
\end{abstract}

\section{INTRODUCTION}

Recently several nonautonomous (with time-dependent coefficients) and inhomogeneous (with space-dependent coefficients) nonlinear Schrödinger equations have been discussed as (possible) new integrable systems [7, 8], 14, 16, 20], 42,

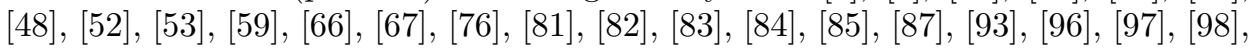
104 (see also [2, 3], 9], 18, 19], 21, 45], 55], 92] and the references therein for earlier works). They arise in the theory of Bose-Einstein condensation [35, 75], fiber optics 6, 47, superconductivity and plasma physics [18, 19, 70, 71.

As pointed out in recent papers [7, [16, [48, [49] and [55] (see also [2], 3], 21, [34, 45], 68, 74]), these systems can be reduced by a set of transformations to the standard autonomous nonlinear Schrödinger equation, which explains their integrability properties, because this equation is a well-known complete integrable system with Lax pair [58, [101, 102, [103, conservation laws and $N$-soliton solutions, solvable through the inverse scattering method [2, 3], [5], [54, [71, [78, 101], 102, [103. Integration techniques of the nonlinear Schrödinger equation also include Painlevé analysis [13, 24, 25], 26, 27, [33, [34, 49], [54, 69, 89, 54, the Hirota method [50, [51, [54, Bäcklund transformation [12, 17, [54] and the Hamiltonian approach [1], 5], 43], 44, 64, 65], 71], among others [36, 62], 72, 77.

A goal of this paper is to construct these transformations explicitly (in quadratures) for the most general variable quadratic Hamiltonian. A simple relation with Green's function of the linear problem, which seems to be missing in the available literature, is emphasized. Basics of the classical soliton theory, including one and two soliton solutions, the inverse scattering technique and the corresponding equivalent Lax pair, are also briefly summarized in order to make our presentation as

Received by the editors March 18, 2011.

2010 Mathematics Subject Classification. Primary 35Q55, 35Q51; Secondary 35P30, 81Q05.

Key words and phrases. Nonlinear Schrödinger equations, generalized harmonic oscillators, Green's function, propagator, completely integrable systems, Lax pair, Zakharov-Shabat system. 
self-contained as possible. This summary may facilitate applications of our result to specific nonlinear integrable systems.

\section{TRANSFORMATION INTO AUTONOMOUS FORM}

We consider the nonautonomous nonlinear Schrödinger equation

$$
i \frac{\partial \psi}{\partial t}=H \psi+h|\psi|^{2} \psi
$$

on $\mathbb{R}$, where the variable Hamiltonian $H=Q(p, x, t)$ is an arbitrary quadratic form of operators $p=-i \partial / \partial x$ and $x$; namely,

$i \psi_{t}=-a(t) \psi_{x x}+b(t) x^{2} \psi-i c(t) x \psi_{x}-i d(t) \psi-f(t) x \psi+i g(t) \psi_{x}+h(t)|\psi|^{2} \psi$ $(a, b, c, d, f$ and $g$ are suitable real-valued functions of time only) under the following integrability condition 87,1$]$

$$
h=h_{0} a(t) \beta^{2}(t) \mu(t)=h_{0} \beta^{2}(0) \mu^{2}(0) \frac{a(t) \lambda^{2}(t)}{\mu(t)}
$$

( $h_{0}$ is a real constant, and functions $\beta, \lambda$ and $\mu$ will be defined below).

We present the following result.

Lemma 1. The substitution

$$
\psi(x, t)=\frac{1}{\sqrt{\mu(t)}} e^{i\left(\alpha(t) x^{2}+\delta(t) x+\kappa(t)\right)} \chi(\xi, \tau), \quad \xi=\beta(t) x+\varepsilon(t), \quad \tau=\gamma(t),
$$

transforms the nonautonomous and inhomogeneous nonlinear Schrödinger equation (2.2) into the standard autonomous form with respect to new variables $\xi=\beta(t) x+$ $\varepsilon(t)$ and $\tau=\gamma(t)$ :

$$
i \chi_{\tau}+h_{0}|\chi|^{2} \chi=\chi_{\xi \xi}
$$

provided that

$$
\begin{gathered}
\frac{d \alpha}{d t}+b+2 c \alpha+4 a \alpha^{2}=0, \\
\frac{d \beta}{d t}+(c+4 a \alpha) \beta=0, \\
\frac{d \gamma}{d t}+a \beta^{2}=0
\end{gathered}
$$

and

$$
\begin{gathered}
\frac{d \delta}{d t}+(c+4 a \alpha) \delta=f+2 \alpha g, \\
\frac{d \varepsilon}{d t}=(g-2 a \delta) \beta, \\
\frac{d \kappa}{d t}=g \delta-a \delta^{2},
\end{gathered}
$$

where

$$
\alpha=\frac{1}{4 a(t)} \frac{\mu^{\prime}(t)}{\mu(t)}-\frac{d(t)}{2 a(t)} .
$$

\footnotetext{
${ }^{1}$ If the nonlinear term has the form $h|\psi|^{p} \psi$, popular in the mathematical literature, the condition becomes $h=h_{0} a \beta^{2} \mu^{p / 2}$.
} 
The autonomous equation (2.5) is completely integrable by advanced methods of the soliton theory [5], [54, [71], 79], [101, [102, [103] (see also [36] and the references cited in the introduction). Equations (2.2) -(2.3) seem to represent the maximum nonautonomous and inhomogeneous one-dimensional integrable system of this kind. (Important special cases of the transformation (2.4) are discussed in [2], 18], [19], 21], [45], 49], [55], [74, 76] and [87].)

Our transformation (2.4) involves the real-valued functions $\alpha, \beta, \gamma, \delta, \varepsilon$ and $\kappa$ (of time $t$ only) defined as solutions of the system of ordinary differential equations (2.6) -(2.11). This nonlinear ODE system has already appeared in 28, from a different perspective (we shall refer to this system as a Riccati-type system). The substitution (2.12) reduces the Riccati equation (2.6) to the second order linear equation [28]:

$$
\mu^{\prime \prime}-\tau(t) \mu^{\prime}+4 \sigma(t) \mu=0
$$

with

$$
\tau(t)=\frac{a^{\prime}}{a}-2 c+4 d, \quad \sigma(t)=a b-c d+d^{2}+\frac{d}{2}\left(\frac{a^{\prime}}{a}-\frac{d^{\prime}}{d}\right) .
$$

(Relations with the corresponding Ehrenfest theorem for the linear Hamiltonian are discussed in [30. It provides a clear physical interpretation of our results in the case of the Gross-Pitaevskii model of Bose condensation.) Equation (2.8) implies the monotonicity of the new time variable $\tau=\gamma(t)$.

Proof. Differentiate $\psi=\mu^{-1 / 2}(t) e^{i S(x, t)} \chi(\xi, \tau)$ with $S=\alpha(t) x^{2}+\delta(t) x+\kappa(t)$ and $\xi=\beta(t) x+\varepsilon(t), \tau=\gamma(t)$ :

$$
\begin{gathered}
i e^{-i S} \psi_{t}=\frac{1}{\sqrt{\mu}}\left[-\left(\alpha^{\prime} x^{2}+\delta^{\prime} x+\kappa^{\prime}\right) \chi+i\left(\left(\beta^{\prime} x+\varepsilon^{\prime}\right) \chi_{\xi}+\gamma^{\prime} \chi_{\tau}-\frac{\mu^{\prime}}{2 \mu} \chi\right)\right] \\
e^{-i S} \psi_{x}=\frac{1}{\sqrt{\mu}}\left[i(2 \alpha x+\delta) \chi+\beta \chi_{\xi}\right]
\end{gathered}
$$

and

$$
e^{-i S} \psi_{x x}=\frac{1}{\sqrt{\mu}}\left[\left(2 i \alpha-(2 \alpha x+\delta)^{2}\right) \chi+2 i(2 \alpha x+\delta) \beta \chi_{\xi}+\beta^{2} \chi_{\xi \xi}\right] .
$$

Substitution into (2.2), with the help of the integrability condition (2.3) and the system (2.6)-(2.11), results in (2.5). Computational details are left to the reader.

This observation provides a new interpretation of the Riccati-type system (2.6)(2.11), which was originally derived in [28] during integration of the corresponding linear equation via Green's function.

\section{INTEGRATION OF THE RicCATI-TYPE SYSTEM}

In order to construct the transformation (2.4) explicitly, one has to solve the nonlinear ODE system (2.6) (2.11). As already known, the initial value problem of the Riccati-type system, which corresponds to the linear Schrödinger equation with a variable quadratic Hamiltonian (generalized harmonic oscillators [11, 40], 46], [95, [100]), can be explicitly solved in terms of solutions of characteristic equation (2.13) as follows (28, [30, 86, 88]):

$$
\mu(t)=2 \mu(0) \mu_{0}(t)\left(\alpha(0)+\gamma_{0}(t)\right)
$$




$$
\begin{aligned}
& \alpha(t)=\alpha_{0}(t)-\frac{\beta_{0}^{2}(t)}{4\left(\alpha(0)+\gamma_{0}(t)\right)}, \\
& \beta(t)=-\frac{\beta(0) \beta_{0}(t)}{2\left(\alpha(0)+\gamma_{0}(t)\right)}=\frac{\beta(0) \mu(0)}{\mu(t)} \lambda(t), \\
& \gamma(t)=\gamma(0)-\frac{\beta^{2}(0)}{4\left(\alpha(0)+\gamma_{0}(t)\right)}
\end{aligned}
$$

and

$$
\begin{aligned}
\delta(t) & =\delta_{0}(t)-\frac{\beta_{0}(t)\left(\delta(0)+\varepsilon_{0}(t)\right)}{2\left(\alpha(0)+\gamma_{0}(t)\right)}, \\
\varepsilon(t) & =\varepsilon(0)-\frac{\beta(0)\left(\delta(0)+\varepsilon_{0}(t)\right)}{2\left(\alpha(0)+\gamma_{0}(t)\right)}, \\
\kappa(t) & =\kappa(0)+\kappa_{0}(t)-\frac{\left(\delta(0)+\varepsilon_{0}(t)\right)^{2}}{4\left(\alpha(0)+\gamma_{0}(t)\right)} .
\end{aligned}
$$

Here,

$$
\begin{aligned}
& \alpha_{0}(t)=\frac{1}{4 a(t)} \frac{\mu_{0}^{\prime}(t)}{\mu_{0}(t)}-\frac{d(t)}{2 a(t)}, \\
& \beta_{0}(t)=-\frac{\lambda(t)}{\mu_{0}(t)}, \quad \lambda(t)=\exp \left(-\int_{0}^{t}(c(s)-2 d(s)) d s\right), \\
& \gamma_{0}(t)=\frac{1}{2 \mu_{1}(0)} \frac{\mu_{1}(t)}{\mu_{0}(t)}+\frac{d(0)}{2 a(0)}
\end{aligned}
$$

and

$$
\begin{aligned}
\delta_{0}(t)= & \frac{\lambda(t)}{\mu_{0}(t)} \int_{0}^{t}\left[\left(f(s)-\frac{d(s)}{a(s)} g(s)\right) \mu_{0}(s)+\frac{g(s)}{2 a(s)} \mu_{0}^{\prime}(s)\right] \frac{d s}{\lambda(s)}, \\
\varepsilon_{0}(t)= & -\frac{2 a(t) \lambda(t)}{\mu_{0}^{\prime}(t)} \delta_{0}(t)+8 \int_{0}^{t} \frac{a(s) \sigma(s) \lambda(s)}{\left(\mu_{0}^{\prime}(s)\right)^{2}}\left(\mu_{0}(s) \delta_{0}(s)\right) d s \\
& +2 \int_{0}^{t} \frac{a(s) \lambda(s)}{\mu_{0}^{\prime}(s)}\left(f(s)-\frac{d(s)}{a(s)} g(s)\right) d s \\
\kappa_{0}(t)= & \frac{a(t) \mu_{0}(t)}{\mu_{0}^{\prime}(t)} \delta_{0}^{2}(t)-4 \int_{0}^{t} \frac{a(s) \sigma(s)}{\left(\mu_{0}^{\prime}(s)\right)^{2}}\left(\mu_{0}(s) \delta_{0}(s)\right)^{2} d s \\
& -2 \int_{0}^{t} \frac{a(s)}{\mu_{0}^{\prime}(s)}\left(\mu_{0}(s) \delta_{0}(s)\right)\left(f(s)-\frac{d(s)}{a(s)} g(s)\right) d s
\end{aligned}
$$

$\left(\delta_{0}(0)=-\varepsilon_{0}(0)=g(0) /(2 a(0))\right.$ and $\left.\kappa_{0}(0)=0\right)$ provided that $\mu_{0}$ and $\mu_{1}$ are the standard solutions of equation (2.13) corresponding to the initial conditions $\mu_{0}(0)=0, \mu_{0}^{\prime}(0)=2 a(0) \neq 0$ and $\mu_{1}(0) \neq 0, \mu_{1}^{\prime}(0)=0$ (proofs are outlined in [28, 32 and [86]). (Formulas (3.8)-(3.13) correspond to Green's function of generalized harmonic oscillators; see, for example, [28, [30, 41], [56], 60, [86, [88] and the references therein for more details.)

One may refer to the solutions (3.8)-(3.13) as the fundamental solution of the Riccati-type system (2.6)-(2.11). Thus the transformation property (3.1)-(3.7) allows one to find a solution of the initial value problem in terms of the fundamental solution (for the nonlinear ODE system). 


\section{INTEGRATION OF THE NONAUTONOMOUS LINEAR SYSTEM}

The transformation (2.4) reduces the linear Schrödinger equation of generalized harmonic oscillators, namely, equation (2.1) with $h=0$, to the Schrödinger equation for a free particle $i \chi_{\tau}=\chi_{\xi \xi}$ with a familiar Green function given by

$$
G\left(\xi, \eta, \tau-\tau_{0}\right)=\frac{1}{\sqrt{-4 \pi i\left(\tau-\tau_{0}\right)}} \exp \left[-i \frac{(\xi-\eta)^{2}}{4\left(\tau-\tau_{0}\right)}\right],
$$

where $\xi=\beta(t) x+\varepsilon(t), \eta=\beta(0) x+\varepsilon(0)$ and $\tau=\gamma(t), \tau_{0}=\gamma(0)$. One can verify directly that Green's functions of generalized harmonic oscillators [28],

$$
\begin{aligned}
G(x, y, t)=\frac{1}{\sqrt{2 \pi i \mu_{0}(t)}} \exp \left[i \left(\alpha_{0}(t) x^{2}+\beta_{0}(t) x y\right.\right. & +\gamma_{0}(t) y^{2} \\
& \left.\left.+\delta_{0}(t) x+\varepsilon_{0}(t) y+\kappa_{0}(t)\right)\right],
\end{aligned}
$$

are derived from the simplest free particle propagator 4.1 with the help of our transformations (3.1)-(3.7). In is worth noting, though, that the transformation (2.4) requires a knowledge of the functions $\mu, \alpha, \beta, \gamma, \delta, \varepsilon$ and $\kappa$, which allows us to determine Green's function for the generalized harmonic oscillators directly from (2.2). Thus finding this transformation is equivalent to integration of the original linear equation from the very beginning. (Lemma 1 extends this observation to the nonlinear Schrödinger equation (2.2).)

Then the superposition principle allows us to solve the corresponding Cauchy initial value problem:

$$
\psi(x, t)=\int_{-\infty}^{\infty} G(x, y, t) \psi(y, 0) d y
$$

for suitable initial data $\psi(x, 0)=\varphi(x)$ (see [28, 88] and [86] for details).

As shown in [86, the following asymptotics hold:

$$
\begin{aligned}
& \alpha_{0}(t)=\frac{1}{4 a(0) t}-\frac{c(0)}{4 a(0)}-\frac{a^{\prime}(0)}{8 a^{2}(0)}+\mathcal{O}(t), \\
& \beta_{0}(t)=-\frac{1}{2 a(0) t}+\frac{a^{\prime}(0)}{4 a^{2}(0)}+\mathcal{O}(t), \\
& \gamma_{0}(t)=\frac{1}{4 a(0) t}+\frac{c(0)}{4 a(0)}-\frac{a^{\prime}(0)}{8 a^{2}(0)}+\mathcal{O}(t), \\
& \delta_{0}(t)=\frac{g(0)}{2 a(0)}+\mathcal{O}(t), \quad \varepsilon_{0}(t)=-\frac{g(0)}{2 a(0)}+\mathcal{O}(t), \\
& \kappa_{0}(t)=\mathcal{O}(t)
\end{aligned}
$$

as $t \rightarrow 0$ for sufficiently smooth coefficients. Then

$$
\begin{aligned}
G(x, y, t) & \sim \frac{1}{\sqrt{2 \pi i a(0) t}} \exp \left[i \frac{(x-y)^{2}}{4 a(0) t}\right] \\
& \times \exp \left[-i\left(\frac{a^{\prime}(0)}{8 a^{2}(0)}(x-y)^{2}+\frac{c(0)}{4 a(0)}\left(x^{2}-y^{2}\right)-\frac{g(0)}{2 a(0)}(x-y)\right)\right]
\end{aligned}
$$

as $t \rightarrow 0$, which corrects a typo in [28]. (Here, $f \sim g$ as $t \rightarrow 0$, if $\lim _{t \rightarrow 0}(f / g)=1$.) 
Another form of solution of the linear problem can be found by an eigenfunction expansion [56], 88].

Numerous examples of (super) integrable (driven) generalized harmonic oscillators with a detailed bibliography can be found, for instance, in recent publications 28, 29, 30, 31 and 32. In addition, our Lemma 1 shows that these results provide explicit transformations (2.4) of the corresponding nonlinear systems into the standard completely integrable forms.

\section{ONE SOLITON SOLUTION}

In the next few sections, we summarize basics of the inverse scattering technique for the autonomous nonlinear Schrödinger equation (2.5) in order to facilitate use of the transformation (2.4) for specific nonautonomous and inhomogeneous nonlinear Schrödinger equations (2.2) from various applications. More details, when needed, can be found in classical works [2], [5], [43, [71, [79], 101, [102, [103] (see also the references cited in the introduction).

As is well-known, equation (2.5) has a traveling wave solution of the form

$$
\chi(\xi, \tau)=e^{i\left(\xi y+\tau\left(y^{2}-g_{0}\right)+\phi\right)} F(\xi+2 \tau y)
$$

provided

$$
\left(\frac{d F}{d z}\right)^{2}=C_{0}+g_{0} F^{2}+\frac{1}{2} h_{0} F^{4} \quad\left(C_{0} \text { is a constant of integration }\right) .
$$

Examples include bright and dark solitons, as well as Jacobi elliptic transcendental solutions for nonlinear wave profiles [2, [54, 71], [79], 87]. Setting $C_{0}=y=0$ gives the stationary breather, which is located about $\xi=0$ and oscillates at a frequency equal to $g_{0}$ 78, 79].

By (2.4), the nonautonomous Schrödinger equation (2.2) under the integrability condition (2.3) has the following solution:

$$
\begin{aligned}
\psi(x, t)= & \frac{e^{i \phi}}{\sqrt{\mu}} \exp \left(i\left(\alpha x^{2}+\beta x y+\gamma\left(y^{2}-g_{0}\right)+\delta x+\varepsilon y+\kappa\right)\right) \\
& \times F(\beta x+2 \gamma y+\varepsilon),
\end{aligned}
$$

where the elliptic function $F$ satisfies equation (5.2) and $\phi, y, g_{0}$ and $h_{0}$ are real parameters (see also [87] for a direct derivation of this solution).

\section{Integrability of NONAUtonomous NONLINEAR SChrÖDINGER EQUATIONS}

The substitution $\Psi(X, T)=\sqrt{h_{0}} \chi(\sqrt{2} X,-2 T)$ transforms equation (2.5) into the standard forms

$$
i \Psi_{T}+\Psi_{X X} \pm 2|\Psi|^{2} \Psi=0
$$

(focusing and defocusing), which can be obtained as the flatness condition

$$
U_{T}-V_{X}+U V-V U=0
$$

for the Lax-(Zakharov-Shabat) pair

$$
\begin{aligned}
U & =-i \lambda \sigma_{3}+\Psi \sigma_{+} \mp \Psi^{*} \sigma_{-} \\
& =\left(\begin{array}{cc}
-i \lambda & \Psi \\
\mp \Psi^{*} & i \lambda
\end{array}\right)
\end{aligned}
$$


and

$$
\begin{aligned}
V & =i\left(-2 \lambda^{2} \pm|\Psi|^{2}\right) \sigma_{3}+\left(2 \lambda \Psi+i \Psi_{X}\right) \sigma_{+} \pm\left(-2 \lambda \Psi^{*}+i \Psi_{X}^{*}\right) \sigma_{-} \\
& =\left(\begin{array}{cc}
i\left(-2 \lambda^{2} \pm|\Psi|^{2}\right) & 2 \lambda \Psi+i \Psi_{X} \\
\mp 2 \lambda \Psi^{*} \pm i \Psi_{X}^{*} & i\left(2 \lambda^{2} \mp|\Psi|^{2}\right.
\end{array}\right)
\end{aligned}
$$

(we use the asterisk for complex conjugation). Here, $\lambda$ is a constant, $\sigma_{ \pm}=$ $\left(\sigma_{1} \pm i \sigma_{2}\right) / 2$ and $\sigma_{1}, \sigma_{2}, \sigma_{3}$ are the Pauli matrices

$$
\sigma_{1}=\left(\begin{array}{cc}
0 & 1 \\
1 & 0
\end{array}\right), \quad \sigma_{2}=\left(\begin{array}{cc}
0 & -i \\
i & 0
\end{array}\right), \quad \sigma_{3}=\left(\begin{array}{cc}
1 & 0 \\
0 & -1
\end{array}\right) .
$$

Since the Lax pair guarantees complete integrability and can alone derive all its associated properties, our transformation (2.4) trivially explains the integrability features of the nonautonomous nonlinear Schrödinger equation (2.1), including $N$-soliton solutions, infinite conservative properties, etc. (see [55], 71] and [79] for more details).

\section{INVERSE SCATTERING METHOD}

The solution of the Cauchy initial value problem through the inverse scattering method is discussed in [3], [5], [54, 71], [79], 101], 102] and [103]. In the focusing case,

$$
i \Psi_{T}+\Psi_{X X}+2|\Psi|^{2} \Psi=0
$$

Zakharov-Shabat's system contains four equations for an auxiliary two-component wave function $\boldsymbol{\Phi}=(\varphi, v)^{T}$ :

$$
\boldsymbol{\Phi}_{X}=U \boldsymbol{\Phi}, \quad \boldsymbol{\Phi}_{T}=V \boldsymbol{\Phi}
$$

namely,

$$
\begin{aligned}
& \varphi_{X}=-i \lambda \varphi+\Psi v \\
& v_{X}=-\Psi^{*} \varphi+i \lambda v
\end{aligned}
$$

and

$$
\begin{aligned}
& \varphi_{T}=i\left(-2 \lambda^{2}+|\Psi|^{2}\right) \varphi+\left(2 \lambda \Psi+i \Psi_{X}\right) v, \\
& v_{T}=\left(-2 \lambda \Psi^{*}+i \Psi_{X}^{*}\right) \varphi+i\left(2 \lambda^{2}-|\Psi|^{2}\right) v .
\end{aligned}
$$

Assuming that $\Psi(X, T) \rightarrow 0$ (and $\left.\Psi_{X}(X, T) \rightarrow 0\right)$ and as $X \rightarrow \pm \infty$ implies

$$
\varphi_{T} \rightarrow-2 i \lambda^{2} \varphi, \quad v_{T} \rightarrow 2 i \lambda^{2} v \quad(X \rightarrow \pm \infty),
$$

the scattering data for the problem

$$
\begin{aligned}
& L\left(\begin{array}{c}
\varphi \\
v
\end{array}\right)=\lambda\left(\begin{array}{c}
\varphi \\
v
\end{array}\right), \\
& L=i \sigma_{3} \frac{\partial}{\partial X}-i \Psi \sigma_{+}-i \Psi^{*} \sigma_{-}=i\left(\begin{array}{cc}
\partial_{X} & -\Psi \\
-\Psi^{*} & -\partial_{X}
\end{array}\right)
\end{aligned}
$$

evolved with time as

$$
b(\lambda, T)=b\left(\lambda, T_{0}\right) e^{4 i \lambda^{2}\left(T-T_{0}\right)}, \quad r_{n}(T)=r_{n}\left(T_{0}\right) e^{4 i \lambda\left(T-T_{0}\right)} .
$$


Then the Cauchy initial value problem for the nonlinear Schrödinger equation (6.1) can be solved as follows [54, [79], 101], 102], 103]:

$$
\Psi(X, T)=-2 K(X, X, T),
$$

where $K(X, Y, T)$ satisfies the linear integral equation

$$
\begin{aligned}
K(X, Y, T)= & B^{*}(X+Y, T) \\
& -\int_{X}^{\infty} \int_{X}^{\infty} K(X, Z, T) B(Z+W, T) B^{*}(Y+W, T) d Z d W
\end{aligned}
$$

and $B(X, T)$ can be obtained in terms of the scattering data:

$$
\begin{aligned}
B(X, T)= & -i \sum_{n=1}^{N} r_{n}\left(T_{0}\right) e^{i\left(\lambda_{n} X+4 \lambda_{n}^{2}\left(T-T_{0}\right)\right)} \\
& +\frac{1}{2 \pi} \int_{-\infty}^{\infty} b\left(\lambda, T_{0}\right) e^{i\left(\lambda X+4 \lambda^{2}\left(T-T_{0}\right)\right)} d \lambda
\end{aligned}
$$

(see [2], 3], [5], 54, 71], [78, 79], 91], 101, 102], 103] for more details).

As a result, the combination of gauge, scaling and coordinate transformations [55],

$$
\psi(x, t)=\frac{1}{\sqrt{h_{0} \mu(t)}} e^{i\left(\alpha(t) x^{2}+\delta(t) x+\kappa(t)\right)} \Psi\left(\frac{1}{\sqrt{2}}(\beta(t) x+\varepsilon(t)),-\frac{1}{2} \gamma(t)\right),
$$

written here explicitly in terms of the solution of the Riccati-type system (2.6)(2.11) from section 3, allows us to solve the Cauchy initial value problem for the nonautonomous nonlinear Schrödinger equation (2.2) with the help of the standard inverse scattering technique. The following choice of parameters, $\alpha(0)=\gamma(0)=$ $\delta(0)=\kappa(0)=\varepsilon(0)=0, \beta(0)=\sqrt{2}, h_{0} \mu(0)=1$, preserves the initial data, $\psi(x, 0)=\Psi(\xi, 0)$, and simplifies the solution (3.1)- (3.7).

\section{TwO SOLITON SOLUTION}

Two well-known solutions of (77.1) are given by [78, [79]:

$$
\Psi_{1}(X, T)=\frac{e^{i T}}{\cosh X}
$$

and

$$
\Psi_{2}(X, T)=4 e^{i T} \frac{\cosh 3 X+3 e^{8 i T} \cosh X}{\cosh 4 X+4 \cosh 2 X+3 \cos 8 T} .
$$

Use of the transformation (7.12) results in one and two soliton solutions for the nonautonomous nonlinear Schrödinger equation (2.2), respectively. (See 78, 79] and 101, for more details. $N$-soliton solutions are also discussed in [2, 62, and 71.)

\section{Transformation of the Lax Pair And Zakharov-Shabat System}

If needed, an equivalent (nonisospectral) Lax pair for the nonautonomous Schrödinger equation (2.2), which is discussed in [9, 18, [19, 21], 84, 85] for important special cases (see also [7] and [16]), can be derived, in general, from (6.3)-(6.4) by inverting our transformation (7.12) (see [3], [55] for more details). In this paper, the required integrability condition (2.3) (found for the soliton-like solution in [87]) 
has been already incorporated into this transformation. Computational details are left to the reader.

\section{EXAMPLES}

A few simple examples are in order. (More examples can be found in 30] and 87]; see also the references therein.)

10.1. Example 1. As noticed by Clarkson [21] (see also [2] and [68]), the equation

$$
i \psi_{t}+\psi_{x x}=\frac{\omega^{2}}{4} x^{2} \psi+2|\psi|^{2} \psi, \quad \omega \neq 0
$$

does not pass the Painlevé test and, therefore, is not integrable. The corresponding characteristic equation, $\mu^{\prime \prime}+\omega^{2} \mu=0$, has two standard solutions:

$$
\mu_{0}=\frac{2}{\omega} \sin \omega t, \quad \mu_{1}=\cos \omega t .
$$

By our Lemma 1, a modified equation for harmonic solitons [45], [87,

$$
i \psi_{t}+\psi_{x x}=\frac{\omega^{2}}{4} x^{2} \psi+\frac{h_{0} \omega \mu(0) \beta^{2}(0)}{4 \alpha(0) \sin \omega t+\omega \cos \omega t}|\psi|^{2} \psi
$$

$\left(h_{0}, \alpha(0), \beta(0) \neq 0\right.$ and $\mu(0)$ are real constants), can be transformed into the standard form and hence is integrable. Here, $\mu(t)=\mu(0)[4 \alpha(0) \sin \omega t+\omega \cos \omega t] / \omega$ and a general solution of the corresponding Riccati-type system is given by

$$
\begin{aligned}
\alpha(t) & =\frac{\omega}{4} \frac{4 \alpha(0) \cos \omega t-\omega \sin \omega t}{4 \alpha(0) \sin \omega t+\omega \cos \omega t}, \\
\beta(t) & =\frac{\omega \beta(0)}{4 \alpha(0) \sin \omega t+\omega \cos \omega t}, \\
\gamma(t) & =\gamma(0)-\frac{\beta^{2}(0) \sin \omega t}{4 \alpha(0) \sin \omega t+\omega \cos \omega t}, \\
\delta(t) & =\frac{\omega \delta(0)}{4 \alpha(0) \sin \omega t+\omega \cos \omega t}, \\
\varepsilon(t) & =\varepsilon(0)-\frac{2 \beta(0) \delta(0) \sin \omega t}{4 \alpha(0) \sin \omega t+\omega \cos \omega t} \\
\kappa(t) & =\kappa(0)-\frac{\delta^{2}(0) \sin \omega t}{4 \alpha(0) \sin \omega t+\omega \cos \omega t}
\end{aligned}
$$

Letting $\mu(0)=\beta(0)=1$ and $\alpha(0)=\gamma(0)=\delta(0)=\varepsilon(0)=\kappa(0)=0$, we arrive at the simple substitution

$$
\psi(x, t)=\frac{e^{-i(\omega / 4) x^{2} \tan \omega t}}{\sqrt{\cos \omega t}} \chi\left(\frac{x}{\cos \omega t},-\frac{\tan \omega t}{\omega}\right),
$$

which transforms (10.3) into (2.5). (Replace $\omega=i \varkappa$ for the original equation in 45].)

In a similar fashion, the equation

$$
i \psi_{t}+\psi_{x x}+\left(k^{2} x^{2}-i k\right) \psi=\frac{2 k h_{0} \mu(0) \beta^{2}(0)}{(k+2 \alpha(0)) e^{4 k t}+k-2 \alpha(0)}|\psi|^{2} \psi
$$


$(k \neq 0, \alpha(0), \beta(0) \neq 0$ and $\mu(0)$ are real constants $)$ is integrable. Indeed, the characteristic equation and the standard solutions are given by

$$
\mu^{\prime \prime}+4 k \mu^{\prime}=0, \quad \mu_{0}=\frac{1}{2 k}\left(1-e^{-4 k t}\right), \quad \mu_{1}=1
$$

and

$$
\mu=\frac{\mu(0)}{2 k}\left[k+2 \alpha(0)+(k-2 \alpha(0)) e^{-4 k t}\right], \quad \lambda=e^{-2 k t} .
$$

The simplest case occurs when $k+2 \alpha(0)=0$ [21]. Further details are left to the reader.

10.2. Example 2. A soliton moving with acceleration in linearly inhomogeneous plasma was discovered in [18] and [19] (see also 9] and 92]). For a modified equation with the integrability condition (2.3),

$$
i \frac{\partial \psi}{\partial t}+\frac{\partial^{2} \psi}{\partial x^{2}}+2 k x \psi=\frac{h_{0} \mu_{0} \beta_{0}^{2}}{1+4 \alpha_{0} t}|\psi|^{2} \psi
$$

where $k, h_{0}, \alpha_{0}, \beta_{0}$ and $\mu_{0}$ are constants, we get $\mu(t)=\mu_{0}\left(1+4 \alpha_{0} t\right)$ and

$$
\begin{array}{lll}
\alpha(t)=\frac{\alpha_{0}}{1+4 \alpha_{0} t}, & \beta(t)=\frac{\beta_{0}}{1+4 \alpha_{0} t}, \\
\gamma(t)=\gamma_{0}-\frac{\beta_{0}^{2} t}{1+4 \alpha_{0} t}, & \delta(t)=k t+\frac{\delta_{0}+k t}{1+4 \alpha_{0} t}, \\
\varepsilon(t)=\varepsilon_{0}-\frac{2 \beta_{0} t\left(\delta_{0}+k t\right)}{1+4 \alpha_{0} t}, & \kappa(t)=\kappa_{0}-\frac{k^{2} t^{3}}{3}-\frac{t\left(\delta_{0}+k t\right)^{2}}{1+4 \alpha_{0} t} .
\end{array}
$$

The classical case [18, [19] corresponds to $\alpha_{0}=0$ and $h_{0} \mu_{0} \beta_{0}^{2}=-2($ with $k \rightarrow-k$ ). The simplest transformation,

$$
\psi(x, t)=e^{i\left(2 k t x-4 k^{2} t^{3} / 3\right)} \chi\left(x-2 k t^{2},-t\right),
$$

when $\alpha=\alpha_{0}=\gamma_{0}=\delta_{0}=\varepsilon_{0}=\kappa_{0}=0$ and $\mu_{0}=\beta=1$, is due to Tappert [18.

10.3. Example 3. The nonlinear Schrödinger equation 87]

$$
i \frac{\partial \psi}{\partial t}+\frac{\partial^{2} \psi}{\partial x^{2}}=\frac{g_{0} \beta_{0}^{2}}{\left(1+4 \alpha_{0} t\right)^{2}} z \psi+\frac{h_{0} \mu_{0} \beta_{0}^{2}}{1+4 \alpha_{0} t}|\psi|^{2} \psi
$$

where

$$
z=\frac{\beta_{0} x+2\left(\gamma_{0}-\left(\beta_{0}^{2}-4 \alpha_{0} \gamma_{0}\right) t\right) y}{1+4 \alpha_{0} t},
$$

with the help of the gauge transformation

$$
\psi=e^{-i f(t)} \chi(x, t), \quad \frac{d f}{d t}=2 g_{0} \beta_{0}^{2} y \frac{\gamma_{0}-\left(\beta_{0}^{2}-4 \alpha_{0} \gamma_{0}\right) t}{\left(1+4 \alpha_{0} t\right)^{3}},
$$

can be transformed into a similar form:

$$
i \chi_{t}+\chi_{x x}-\frac{g_{0} \beta_{0}^{3} x}{\left(1+4 \alpha_{0} t\right)^{3}} \chi=\frac{h_{0} \mu_{0} \beta_{0}^{2}}{1+4 \alpha_{0} t}|\chi|^{2} \chi .
$$

Looking for a traveling wave, we indicate the following soliton-like solution [87, [89]:

$$
\chi(x, t)=\frac{e^{i S(x, t)}}{\sqrt{\left|\mu_{0}\right|\left(1+4 \alpha_{0} t\right)}} g_{0}^{1 / 3} \sqrt{\frac{2}{h_{0}}} A_{k_{0}}\left(g_{0}^{1 / 3} z\right),
$$


where

$$
\begin{aligned}
S(x, t)= & \frac{\alpha_{0} x^{2}+\beta_{0} x y+\left(\gamma_{0}-\left(\beta_{0}^{2}-4 \alpha_{0} \gamma_{0}\right) t\right) y^{2}}{1+4 \alpha_{0} t} \\
& +g_{0} \beta_{0}^{2} t \frac{2 \gamma_{0}-\left(\beta_{0}^{2}-8 \alpha_{0} \gamma_{0}\right) t}{\left(1+4 \alpha_{0} t\right)^{2}} y
\end{aligned}
$$

$\left(\alpha_{0}, \beta_{0}, \gamma_{0}, \delta_{0}, \varepsilon_{0}, \kappa_{0}, \mu_{0}, g_{0}, h_{0}, y\right.$ are real constants), and the soliton profile is defined, as a solution of the second Painlevé equation [89], in terms of the nonlinear Airy function $A_{k_{0}}(\zeta)$ with asymptotics given by

$$
A_{k_{0}}(\zeta)=\left\{\begin{array}{c}
k_{0} \operatorname{Ai}(\zeta), \quad \zeta \rightarrow+\infty \\
r|\zeta|^{-1 / 4} \sin \left(s(\zeta)-\theta_{0}\right)+o\left(|\zeta|^{-1 / 4}\right), \quad \zeta \rightarrow-\infty .
\end{array}\right.
$$

Here, $\operatorname{Ai}(\zeta)$ is Airy function, $-1<k_{0}<1$ provided $k_{0} \neq 0, r^{2}=-\pi^{-1} \ln \left(1-k_{0}^{2}\right)$,

$$
s(\zeta)=\frac{2}{3}|\zeta|^{3 / 2}-\frac{3}{4} r^{2} \ln |\zeta|
$$

and

$$
\theta_{0}=\frac{3}{2} r^{2} \ln 2+\arg \Gamma\left(1-\frac{i}{2} r^{2}\right)+\frac{\pi}{4}\left(1-2 \operatorname{sign}\left(k_{0}\right)\right) .
$$

These asymptotics were found in 4 and 80 , and had been proven rigorously in [38 and [39] (see [5], 10], 22], 23], 25], 27], 90] and the references therein for a study of this nonlinear Airy function).

It is worth noting that, in contrast to the previous case, this $A$-soliton moves with a constant velocity when $\alpha_{0}=0$ (notice that the external field has essentially changed the soliton shape; see 87] for more details).

\section{Conclusion}

We have shown how to transform the nonautonomous and inhomogeneous nonlinear Schrödinger equations (2.2) -(2.3) into the standard autonomous form (2.5) that is completely integrable by the inverse scattering approach. This transformation is explicitly written in terms of Green's function of the corresponding linear problem (generalized harmonic oscillators); see Lemma 1 and equations (3.1)-(3.7) and (3.8) (3.13). Combination of these advances allows one to solve the Cauchy initial value problem for the generic nonautonomous integrable quantum system under consideration. Simple examples are considered, and further examples can be found elsewhere; see, for instance, [30] and [87] and the references therein.

\section{ACKNOWLEDGMENTS}

The author thanks George E. Andrews, Carlos Castillo-Chávez, Robert Conte, Vladimir I. Man'ko and Svetlana Roudenko for support, valuable discussions and encouragement. The author is grateful to Nathan Lanfear and Erwin Suazo for help. This work was supported in part by the National Science FoundationEnhancing the Mathematical Sciences Workforce in the 21st Century (EMSW21), award \#0838705; the Alfred P. Sloan Foundation-Sloan National Pipeline Program 
in the Mathematical and Statistical Sciences, award \#LTR 05/19/09; and the National Security Agency-Mathematical \& Theoretical Biology Institute, Research Program for Undergraduates, award \#H98230-09-1-0104.

\section{REFERENCES}

[1] M. J. Ablowitz, D. J. Kaup, A. C. Newell and H. Segur, Nonlinear-evolution equations of physical significance, Phys. Rev. Lett. 31 (1973) \#2, 125-127. MR0406176 (53:9968)

[2] M. Ablowitz and P. A. Clarkson, Solitons, Nonlinear Evolution Equations and Inverse Scattering, Cambridge Univ. Press, Cambridge, 1991. MR1149378 (93g:35108)

[3] M. Ablowitz, B. Prinari and A. D. Trubatch, Discrete and Continuous Schrödinger Systems, Cambridge Univ. Press, Cambridge, 2004. MR2040621 (2005c:37117)

[4] M. Ablowitz and H. Segur, Exact linearization of a Painlevé transcendent, Phys. Rev. Lett. 38 (1977) \#20, 1103-1106. MR0442981 (56:1356)

[5] M. Ablowitz and H. Segur, Solitons and the Inverse Scattering Transform, SIAM, Philadelphia, 1981. MR642018 (84a:35251)

[6] G. P. Agrawal, Nonlinear Fiber Optics, Forth Edition, Academic Press, New York, 2007.

[7] U. Al Khawaja, A comparative analysis of Painlevé, Lax pair, and similarity transformation methods in obtaining the integrability conditions of nonlinear Schrödinger equations, J. Math. Phys. 51 (2010), 053506 (11 pages). MR2666984 (2011b:37138)

[8] R. Atre, P. K. Panigrahi and G. S. Agarwal, Class of solitary wave solutions of the onedimensional Gross-Pitaevskii equation, Phys. Rev. E 73 (2006), 056611. MR2242605 (2007c:82007)

[9] R. Balakrishnan, Soliton propagation in nonunivorm media, Phys. Rev. A 32 (1985) \#2, 1144-1149.

[10] A. P. Bassom, P. A. Clarkson, C. K. Law and J. B. McLeod, Application of uniform asymptotics to the second Painlevé transcendent, Arch. Rat. Mech. Anal. 103 (1998), 241-271. MR.1650002 (99g:34122)

[11] M. V. Berry, Classical adiabatic angles and quantum adiabatic phase, J. Phys. A: Math. Gen. 18 (1985) \#1, 15-27. MR777620 (86g:81034)

[12] M. Boiti and T. Guizhang, Bäcklund transformations via gauge transformations, Nuovo Cimento B 71 (1982) \#2, 253-264. MR685100 (84c:58001)

[13] M. Boiti and F. Pempinelli, Nonlinear Schrödinger equation, Bäcklund transformations and Painlevé transcendents, Nuovo Cimento B 59 (1980) \#1, 40-58. MR.598413 (82g:35098)

[14] J. Bolmonte-Beitia, V. M. Pérez-García, V. Vekslerchik and V. V. Konotop, Localized nonlinear waves in systems with time- and space-modulated nonlinearities, Phys. Rev. Lett. 100 (2008), 164102 (4 pages).

[15] K. Bongs and K. Sengstock, Physics with coherent matter waves, Rep. Prog. Phys. 67 (2004), 907-963.

[16] T. Brugarino and M. Sciacca, Integrability of an inhomogeneous nonlinear Schrödinger equation in Bose-Einstein condensates and fiber optics, J. Math. Phys. 51 (2010), 093503 (18 pages). MR 2742827

[17] H.-H. Chen, General deriviation of Bäcklund transformations from inverse scattering problems, Phys. Rev. Lett. 33 (1974) \#15, 925-928. MR0361484 (50:13929)

[18] H.-H. Chen and Ch.-Sh. Liu, Solitons in nonuniform media, Phys. Rev. Lett. 37 (1976) \#11, 693-697. MR0411488 (53:15222)

[19] H.-H. Chen and Ch.-Sh. Liu, Nonlinear wave and soliton propagation in media with arbitrary inhomogeneities, Phys. Fluids 21 (1978) \#3, 377-380.

[20] Sh. Chen and L. Yi, Chirped self-similar solitons of a generalized Schrödinger equation model, Phys. Rev. E 61 (2005), 016606 (4 pages).

[21] P. A. Clarkson, Painlevé analysis for the damped, driven nonlinear Schrödinger equation, Proc. Roy. Soc. Edin. 109A (1988), 109-126. MR952332 (89k:35200)

[22] P. A. Clarkson, Painlevé transcendents, in: NIST Handbook of Mathematical Functions (F. W. J. Olwer, D. M. Lozier et al., eds.), Cambridge Univ. Press, 2010; see also http://dlmf.nist.gov/32. MR 2723248

[23] P. A. Clarkson and J. B. McLeod, A connection formula for the second Painlevé transcendent, Arch. Rat. Mech. Anal. 103 (1988), 97-138. MR946971 (89e:34010) 
[24] R. Conte, Invariant Painlevé analysis of partial differential equations, Phys. Lett. A 140 (1989) \#7-8, 383-390. MR1020742 (90m:58229)

[25] R. Conte, The Painlevé approach to nonlinear ordinary differential equations, in: The Painlevé Property, One Century Later, CRM Series in Mathematical Physics (R. Conte, ed.), Springer-Verlag, New York, 1999, pp. 77-180. MR.1713577(2000h:34014)

[26] R. Conte, A. P. Fordy and A. Pickering, A perturbative Painlevé approach to nonlinear differential equations, Physica D 69 (1993), 33-58. MR 1245654 (94k:35005)

[27] R. Conte and M. Musette, Elliptic general analytic solutions, Studies in Applied Mathematics 123 (2009) \#1, 63-81. MR2538286 (2010m:35053)

[28] R. Cordero-Soto, R. M. Lopez, E. Suazo and S. K. Suslov, Propagator of a charged particle with a spin in uniform magnetic and perpendicular electric fields, Lett. Math. Phys. 84 (2008) \#2-3, 159-178. MR2415547 (2009m:81055)

[29] R. Cordero-Soto, E. Suazo and S. K. Suslov, Models of damped oscillators in quantum mechanics, Journal of Physical Mathematics 1 (2009), S090603 (16 pages).

[30] R. Cordero-Soto, E. Suazo and S. K. Suslov, Quantum integrals of motion for variable quadratic Hamiltonians, Ann. Phys. 325 (2010) \#9, 1884-1912. MR2718565

[31] R. Cordero-Soto and S. K. Suslov, Time reversal for modified oscillators, Theoretical and Mathematical Physics 162 (2010) \#3, 286-316. MR2682129 (2011h:81100)

[32] R. Cordero-Soto and S. K. Suslov, The degenerate parametric oscillator and Ince's equation, J. Phys. A: Math. Theor. 44 (2011) \#1, 015101 (9 pages). MR2749099

[33] C. M. Cosgrove, Painlevé classification of all semilinear partial differential equations of the second order. I. Hyperbolic equations in two independent variables, Studies in Applied Mathematics 89 (1993) \#1, 1-61. MR.1213884 (94g:35010)

[34] C. M. Cosgrove, Painlevé classification of all semilinear partial differential equations of the second order. II. Parabolic and higher dimentional equations, Studies in Applied Mathematics 89 (1993) \#2, 95-151. MR1219303 (94g:35011)

[35] F. Dalfovo, S. Giorgini, L. P. Pitaevskii and S. Stringari, Theory of Bose-Einstein condensation in trapped gases, Rev. Mod. Phys. 71 (1999), 463-512.

[36] A. Desgasperis, Resource letter Sol-1: Solitons, Am. J. Phys. 66 (1998) 6, 486-497.

[37] A. Desgasperis, Integrable models in nonlinear optics and soliton solutions, J. Phys. A: Math. Theor. 43 (2010), 434001 (18 pages). MR2727775

[38] P. Deift and X. Zhou, A steepest descent method for Riemann-Hilbert problems, Ann. Math. (2) 137 (1993), 295-368. MR1207209 (94d:35143)

[39] P. Deift and X. Zhou, Asymptotics for Painlevé II equation, Comm. Pure Appl. Math. 48 (1995), 227-337. MR:1322812 (96d:34004)

[40] V. V. Dodonov, I. A. Malkin and V. I. Man'ko, Integrals of motion, Green functions, and coherent states of dynamical systems, Int. J. Theor. Phys. 14 (1975) \#1, 37-54. MR0403460 (53:7271)

[41] V. V. Dodonov and V. I. Man'ko, Invariants and correlated states of nonstationary quantum systems, in: Invariants and the Evolution of Nonstationary Quantum Systems, Proceedings of Lebedev Physics Institute, vol. 183, pp. 71-181, Nauka, Moscow, 1987 [in Russian]; English translation published by Nova Science, Commack, New York, 1989, pp. 103-261. MR.937883 (89i:81044)

[42] A. Ebaid and S. M. Khaled, New types of exact solutions for nonlinear Schrödinger equation with cubic nonlinearity, Journal of Computational and Applied Mathematics 235 (2011) \#8, 1984-1992. MR2763119

[43] L. D. Faddeev and L. A. Takhtajan, Hamiltonian Methods in the Theory of Solitons, Springer-Verlag, Berlin, New York, 1987. MR905674 (89m:58103)

[44] C. S. Gardner, J. M. Green, M. D. Kruskai and R. M. Miura, Method for solving the Korteweg-deVries equation, Phys. Rev. Lett. 19 (1967) \#19, 1095-1097.

[45] L. Gagnon and P. Winternitz, Symmetry classes of variable coefficient nonlinear Schrödinger equations, J. Phys. A: Math. Gen. 26 (1993), 7061-7076. MR.1253895 (95f:35238)

[46] J. H. Hannay, Angle variable holonomy in adiabatic excursion of an integrable Hamiltonian, J. Phys. A: Math. Gen 18 (1985) \#2, 221-230. MR784910 (86f:70012)

[47] A. Hasegawa, Optical Solitons in Fibers, Springer-Verlag, Berlin, 1989.

[48] J. He and Y. Li, Designable integrability of the variable coefficient nonlinear Schrödinger equations, Studies in Applied Mathematics 126 (2011) \#3 (15 pages). MR2724563 
[49] X-G. He, D. Zhao, L. Lee and H-G. Luo, Engineering integrable nonautonomous Schrödinger equations, Phys. Rev. E 79 (2009), 056610 (9 pages).

[50] R. Hirota, Exact solution of the Korteweg-de Vries equation for multiple collisions of solitons, Phys. Rev. Lett. 27 (1971) \#18, 1192-1194.

[51] R. Hirota, The Direct Method in Soliton Theory, Cambridge University Press, Cambridge, 2004. MR2085332 (2005f:37137)

[52] Y. I. Kruglov, A. C. Peacock and J. D. Harvey, Exact self-similar solutions of the generalized nonlinear Schrödinger equation with distributed coefficients, Phys. Rev. Lett. 90 (2003) \#11, 113902 (4 pages).

[53] Y. I. Kruglov, A. C. Peacock and J. D. Harvey, Exact solutions of the generalized Schrödinger equation with distributed coefficients, Phys. Rev. E 71 (2005), 1056619 (11 pages).

[54] N. A. Kudryashov, Methods of Nonlinear Mathematical Physics, Intellect, Dolgoprudny, 2010 [in Russian].

[55] A. Kundu, Integrable nonautonomous Schrödinger equations are equivalent to the standard autonomous equation, Phys. Rev. E 79 (2009), 015601(R) (4 pages). MR2552199 (2010i:35375)

[56] N. Lanfear, R. M. Lopez and S. K. Suslov, Exact wave functions for generalized harmonic oscillators, Journal of Russian Laser Research 32 (2011) \#4, 312-321; see also arXiv:11002.5119v1 [math-ph], 24 Feb 2011.

[57] N. Lanfear and S. K. Suslov, The time-dependent Schrödinger equation, Riccati equation and Airy functions, arXiv:0903.3608v5 [math-ph], 22 Apr 2009.

[58] P. D. Lax, Integrals of nonlinear equations of evolution and solitary waves, Commun. Pure Appl. Math. 21 (1968) \#5, 467-490. MR0235310 (38:3620)

[59] Z. X. Liang, Z. D. Zhang and W. M. Liu, Dynamics of a bright solution in Bose-Einstein condensates with time-dependent atomic scattering length in an expulsive parabolic potential, Phys. Rev. Lett. 94 (2005) \#5, 050402 (4 pages).

[60] I. A. Malkin and V. I. Man'ko, Dynamical Symmetries and Coherent States of Quantum System, Nauka, Moscow, 1979 [in Russian]. MR537768 (80k:81001)

[61] I. A. Malkin, V. I. Man'ko and D. A. Trifonov, Linear adiabatic invariants and coherent states, J. Math. Phys. 14 (1973) \#5, 576-582.

[62] V. B. Matveev and M. A. Salle, Darboux Transformation and Solitons, Springer-Verlag, Berlin, 1991. MR 1146435 (93d:35136)

[63] M. Meiler, R. Cordero-Soto, and S. K. Suslov, Solution of the Cauchy problem for a timedependent Schrödinger equation, J. Math. Phys. 49 (2008) \#7, 072102: 1-27. MR.2432026 (2009m:81058)

[64] R. M. Miura, Korteweg-de Vries equation and generalizations. I. A remarkable explicit nonlinear transformation, J. Math. Phys. 9 (1968) \#9, 1202-1204. MR0252825 (40:6042a)

[65] R. M. Miura, C. S. Gardner and M. D. Kruskal, Korteweg-de Vries equation and generalizations. II. Existence of conservation laws and constants of motion, J. Math. Phys. 9 (1968) \#9, 1204-1209. MR0252826 (40:6042b)

[66] J. D. Moores, Nonlinear compression of chirped solitary waves with and without phase modulation, Optics Letters 21 (1996) \#8, 555-557.

[67] J. D. Moores, Oscilatory solitons in a novel integrable model of asynchronomous mode locking, Optics Letters 21 (2001) \#2, 87-89.

[68] M. Musette, Painlevé analysis for nonlinear partial differential equations, in: The Painlevé Property, One Century Later, CRM Series in Mathematical Physics (R. Conte, ed.), Springer-Verlag, New York, 1999. pp. 517-572. MR.1713582 (2000k:37103)

[69] M. Musette and R. Conte, Analytic solitary waves of nonintegrable equations, Physica D 181 (2003), 70-79. MR.2003796 (2004f:35148)

[70] A. C. Newell, Nonlinear tunnelling, J. Math. Phys. 19 (1978) \#5, 1126-1133. MR0484112 (58:4045)

[71] S. Novikov, S. V. Manakov, L. P. Pitaevskii and V. E. Zakharov, Theory of Solitons: The Inverse Scattering Method, Kluwer, Dordrecht, 1984. MR779467 (86k:35142)

[72] P. J. Olver, Applications of Lie Group to Differential Equations, Springer-Verlag, Berlin, 1991. MR 1240056 (94g:58260)

[73] A. N. Oraevsky, Bose condensates from the point of view of laser physics, Quantum Electronics 31 (2001) \#12, 1038-1057. 
[74] V. M. Pérez-García, P. Torres and V. V. Konotop, Similarity transformations for nonlinear Schrödinger equations with time-dependent coefficients, Physica D 221 (2006), 31-36. MR.2259120(2007d:35257)

[75] L. Pitaevskii and S. Stringari, Bose-Einstein Condensation, Oxford University Press, Oxford, 2003. MR2012737(2004i:82038)

[76] S. Ponomarenko and G. P. Agrawal, Optical similaritons in nonlinear waveguides, Optics Letters 32 (2007) \#12, 1659-1661.

[77] C. Rogers and W. K. Scheif, Bäcklund Transformation and Darboux Transformation, Cambridge University Press, Cambridge, 2002.

[78] J. Satsuma and N. Yajima, Initial value problems of one-dimensional self-modulation of nonlinear waves in dispersive media, Supp. Prog. Theor. Phys. 85 (1974), 284-306. MR0463733 $(57: 3675)$

[79] A. Scott, Nonlinear Science: Emergence and Dynamics of Coherent Structures, Second Edition, Oxford University Press, Oxford, 2003. MR.2023425 (2004k:37002)

[80] H. Segur and M. J. Ablowitz, Asymptotic solutions of nonlinear equations and a Painlevé transcendent, Physica D 3 (1981) \#1-2, 165-184.

[81] V. N. Serkin and A. Hasegawa, Novel soliton solutions of the nonlinear Schrödinger equation model, Phys. Rev. Lett. 85 (2000) \#21, 4502-4505.

[82] V. N. Serkin and A. Hasegawa, Soliton management in the nonlinear Schrödinger equation model with varying dispertion, nonlinearity, and gain, JETP Letters 72 (2000) \#2, 89-92.

[83] V. N. Serkin, A. Hasegawa and T. L. Belyeva, Comment on "Exact self-similar solutions of the generalized nonlinear Schrödinger equation with distributed coefficients", Phys. Rev. Lett. 92 (2004) \#19, 199401 (1 page).

[84] V. N. Serkin, A. Hasegawa and T. L. Belyeva, Nonautonomous solitons in external potentials, Phys. Rev. Lett. 98 (2007) \#7, 074102 (4 pages).

[85] V. N. Serkin, A. Hasegawa and T. L. Belyeva, Nonautonomous matter-wave soliton near the Feshbach resonance, Phys. Rev. A 81 (2010), 023610 (19 pages).

[86] E. Suazo and S. K. Suslov, Cauchy problem for Schrödinger equation with variable quadratic Hamiltonians, in preparation.

[87] E. Suazo and S. K. Suslov, Soliton-like solutions for nonlinear Schrödinger equation with variable quadratic Hamiltonians, arXiv:1010.2504v4 [math-ph], 24 Nov 2010.

[88] S. K. Suslov, Dynamical invariants for variable quadratic Hamiltonians, Physica Scripta 81 (2010) \#5, 055006 (11 pp); see also arXiv:1002.0144v6 [math-ph], 11 Mar 2010.

[89] M. Tajiri, Similarity reduction of the one- and two-dimensional nonlinear Schrödinger equations, J. Phys. Soc. Japan 52 (1983) \#2, 1908-1917. MR710726 (84h:35154)

[90] Y. Takei, On exact WKB approach to Ablowitz-Segur's connection problem for the second Painlevé equation, ANZIAM J. 44 (2002), 111-119. MR.1919931 (2003d:34194)

[91] T. Tao, Why are solitons stable?, Bull. Amer. Math. Soc. 46 (2009) \#1, 1-33. MR2457070 (2010b:35390)

[92] F. D. Tappert and N. J. Zabusky, Gradient-induced fission of solitons, Phys. Rev. Lett. 26 (1971) \#26, 1774-1776.

[93] C. Trallero-Giner, J. Drake, V. Lopez-Richard, C. Trallero-Herrero and J. L. Birman, BoseEinstein condensates: Analytical methods for the Gross-Pitaevskii equation, Phys. Lett. A 354 (2006), 115-118.

[94] J. Weiss, M. Tabor and G. Carnevalle, The Painlevé property for partial differential equations, J. Math. Phys. 24 (1983) \#3, 522-526. MR692140 (84c:35101)

[95] K. B. Wolf, On time-dependent quadratic Hamiltonians, SIAM J. Appl. Math. 40 (1981) \#3, 419-431. MR614739 (82f:81033)

[96] Z. Yan, The new extended Jacobian elliptic function expansion algorithm and its applications in nonlinear mathematical physics equations, Computer Physics Communications $\mathbf{1 5 3}$ (2003), 145-154. MR1982750 (2004e:35201)

[97] Z. Yan, An improved algebra method and its applications in nonlinear wave equations, Chaos, Solitons \& Fractals 21 (2004), 1013-1021. MR2042818

[98] Z. Yan, Exact analytical solutions for the generalized non-integrable nonlinear Schrödinger equation with varying coefficients, Phys. Lett. A 374 (2010), 4838-4843. MR.2734517 (2011g:35384) 
[99] Z. Yan and V. V. Konotop, Exact solutions to three-dimensional generalized nonlinear Schrödinger equation with varying potential and nonlinearities, Phys. Rev. E 80 (2009), 036607 (9 pages).

[100] K-H. Yeon, K-K. Lee, Ch-I. Um, T. F. George and L. N. Pandey, Exact quantum theory of a time-dependent bound Hamiltonian systems, Phys. Rev. A 48 (1993) \#4, 2716-2720.

[101] V. E. Zakharov and A. B. Shabat, Exact theory of two-dimensional self-focusing and onedimensional self-modulation of waves in nonlinear media, Zh. Eksp. Teor. Fiz. 61 (1971), 118-134 [Sov. Phys. JETP 34 (1972) \#1, 62-69.] MR0406174 (53:9966)

[102] V. E. Zakharov and A. B. Shabat, A plan for integrating the nonlinear equations of mathematical physics by the method of the inverse scattering problem. I, Functional Analysis and Its Applications 8 (1974) \#3, 226-235 (1975). MR0481668 (58:1768)

[103] V. E. Zakharov and A. B. Shabat, Integration of the nonlinear equations of mathematical physics by the method of the inverse scattering problem. II, Functional Analysis and Its Applications 13 (1979) \#3, 166-174. MR.545363 (82m:35137)

[104] X.-F. Zhang, Q. Yang, J.-F. Zhang, X. Z. Chen and W. M. Liu, Controlling soliton interactions in Bose-Einstein condensates by synchronizing the Feshbach resonance and harmonic trap, Phys. Rev. A 77 (2008), 023613 (7 pages).

School of Mathematical and Statistical Sciences and the Mathematical, Computational and Modeling Sciences Center, Arizona State University, Tempe, Arizona 852871804

E-mail address: sks@asu.edu

URL: http://hahn.la.asu.edu/ ${ }^{\sim}$ suslov/index.html 